\title{
Direct and indirect observations of local deformation properties of saturated sand specimens in undrained cyclic triaxial tests
}

\author{
Junichi Koseki ${ }^{\text {i) }}$, Ryoichiro Hoshino ${ }^{\text {ii)}}$, Yukika Miyashitaiii) and Takeshi Sato ${ }^{\text {iv) }}$
}

\begin{abstract}
i) Professor, Department of Civil Engineering, the University of Tokyo, 7-3-1 Hongo, Bunkyo-ku, Tokyo 113-8656, Japan. ii) Ministry of Land, Infrastructure, Transport and Tourism (formerly graduate student, Dept. of Civil Engineering, Univ. of Tokyo, ditto) iii) Technical support specialist, Institute of Industrial Science, Univ. of Tokyo, 4-6-1 Komaba, Meguro-ku, Tokyo 153-8505, Japan.

iv) Technical director, Integrated Geotechnology Institute Ltd., 1-23-6 Yotuya, Shinjuku-ku, Tokyo 160-0004, Japan
\end{abstract}

\begin{abstract}
A procedure to evaluate directly the local deformation properties of sand specimens was developed by using a transparent membrane and adding colored sand particles to the original ones. By combining it with indirect observation based on the image analysis of the membrane deformation, their comparison was made. The local deformation properties of a dense Toyoura sand specimen that was evaluated by the indirect observation during the liquefaction process in cyclic triaxial test up to a double amplitude axial strain of 5\% were consistent with those evaluated by the direct observation, except for the regions near the top and bottom ends of the specimen.
\end{abstract}

Keywords: liquefaction, triaxial test, image analysis, local deformation

\section{INTRODUCTION}

The deformation of soil specimens in laboratory tests, such as triaxial tests, torsional shear tests and plane strain compression tests, may not be uniform, in particular with heterogeneous specimens as tested by Fauzi et al. (2013) among others. In addition, deformation of homogeneous specimens, such as those of dense sands, may be localized in the post-peak region (Yoshida et al., 1994; Rechenmacher 2003 among others). Under such circumstances, it is required to establish a proper methodology by evaluating their local deformation properties.

In the current study, a procedure to evaluate directly the local deformation properties of sand specimens by using a transparent membrane and adding colored sand particles to the original ones was developed. The local deformation properties of a dense Toyoura sand specimen evaluated by this procedure during the liquefaction process in undrained cyclic triaxial test were compared to those evaluated by the indirect observation based on the image analysis of the membrane deformation.

\section{TEST APPARATUS AND MATERIAL}

A triaxial apparatus with a motor-driven axial loading device was employed. The cylindrical specimen was $75 \mathrm{~mm}$ in diameter and $150 \mathrm{~mm}$ in height. The side view of the specimen was recorded by using a digital camera with a resolution of $4912 * 7360$ pixels.
Toyoura sand with $\mathrm{D}_{50}$ of about $0.2 \mathrm{~mm}$ exhibiting yellowish color as shown in Fig. 1a was used as the test material. In order to make direct observation, blue-colored silica sand particles with $\mathrm{D}_{50}$ of about 0.5 $\mathrm{mm}$ were mixed with the Toyoura sand particles at a mass ratio of $5 \%$. Figure $1 \mathrm{~b}$ shows the coarse blue-colored sand particles before mixing. As the transparent membrane, a silicone-rubber sheet having a thickness of $0.5 \mathrm{~mm}$ was glued into a cylindrical shape (Ishimaru et al., 2011), on which red dots were painted at a spacing of $5 \mathrm{~mm}$ for the purpose of indirect observation, as shown in Fig. 1c.
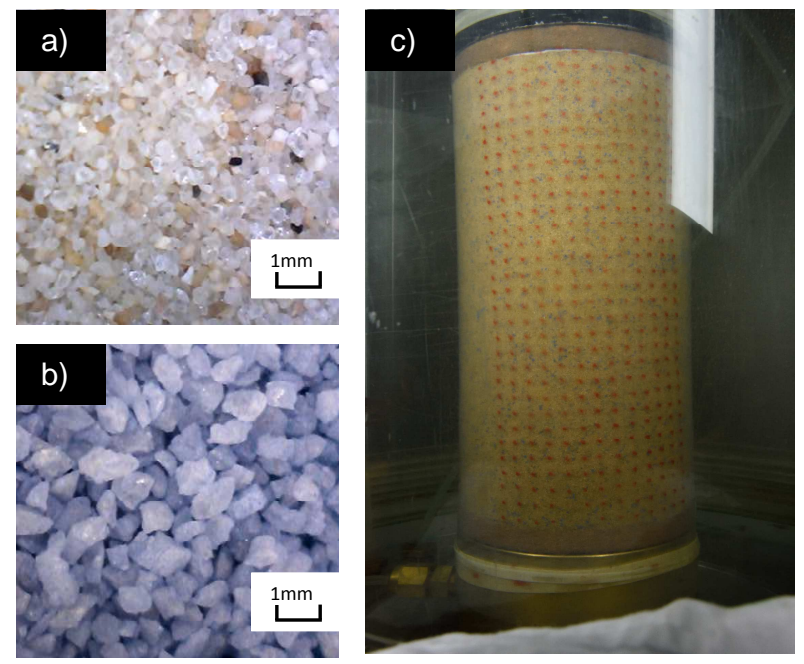

Fig. 1. a) Toyoura sand, b) blue-colored sand particles and c) side view of specimen using transparent membrane with red dots. 


\section{TEST PROCEDURES}

\subsection{Undrained cyclic triaxial test}

The specimen consisted of ten compacted layers and was prepared in a mold by the wet-tamping method, where a pre-determined amount of the mixed material at a water content of $15 \%$ was compacted by using a cylindrical tamper with a diameter of about $15 \mathrm{~mm}$ into the target thickness to achieve an initial relative density in the range of about 70 to $80 \%$. It was frozen in a freezer and then de-molded. After setting the frozen specimen into the triaxial apparatus and applying a confining stress of $30 \mathrm{kPa}$ by using a partial vacuum as the back pressure, it was thawed and then saturated by using the double vacuuming method (Ampadu and Tatsuoka, 1993). Subsequently, it was consolidated isotropically to a confining stress of $100 \mathrm{kPa}$ and subjected to undrained cyclic axial loading with a single amplitude deviator stress of $80 \mathrm{kPa}$.

Since the axial loading system was motor-driven, a constant axial strain rate of $0.1 \% / \mathrm{min}$. was employed for the cyclic loading, and digital photos were taken at an interval of $1 \mathrm{~min}$.

\subsection{Image analysis}

As summarized in Fig. 2, the digital photos were recorded in RAW format originally, which were converted into TIFF (Tagged Image File Format) format for processing the data. In order to contrast the red dots on membrane and blue sand particles, respectively, two sets of image data (to be called as "indirect evaluation data" and "direct evaluation data" herein) were prepared by applying the following filtering process: $\left(\mathrm{R}_{\mathrm{ij}}{ }^{\prime}, \mathrm{G}_{\mathrm{ij}}{ }^{\prime}, \mathrm{B}_{\mathrm{ij}}{ }^{\prime}\right)=\left(255, \mathrm{G}_{\mathrm{ij}}, 255\right)$ for indirect evaluation and $\left(\mathrm{R}_{\mathrm{ij}}, 255,255\right)$ for direct evaluation, where $\left(\mathrm{R}_{\mathrm{ij}}, \mathrm{G}_{\mathrm{ij}}, \mathrm{B}_{\mathrm{ij}}\right)$ and $\left(\mathrm{R}_{\mathrm{ij}}{ }^{\prime}, \mathrm{G}_{\mathrm{ij}}{ }^{\prime}, \mathrm{B}_{\mathrm{ij}}{ }^{\prime}\right)$ are the intensity scale (from 0 to 255 ) of the red, green and blue components before and after the filtering process, respectively. Subsequently, these data sets were converted into gray scale data while enhancing their dynamic range as shown in Fig. 3.

For the indirect and direct evaluation, PIV (Particle image velocimetry)-based analyses of the two sets of data in enhanced gray scale were conducted to trace the movement of the image patterns made by each of the red dots on the membrane and by the group of the blue sand particles, respectively. As shown in Fig. 4a, a region consisting of $71 * 96$ pixels was used to trace each of the red dot patterns for the indirect evaluation. For the direct evaluation, on the other hand, two different regions were used; one region consisted of $168 * 210$ pixels to trace each of the full grid patterns covering the whole area as shown in Fig. $4 \mathrm{~b}$, and the other region consisted of $84 * 84$ pixels that excludes the red dot regions as has been shown in Fig. 4a. Finally, based on the distribution of local displacements evaluated by these analyses, local strain distribution was computed and compared among the indirect and direct evaluation.

It should be noted that, in order to compare between the indirect and direct evaluation results in a simplified manner, no geometric corrections in terms of the distortion of the image caused by cylindrical shape of the specimen as well as the lens effect (Fauzi et al., 2013) were made in this study. For more quantitative assessment of the local deformation, these corrections would be required.

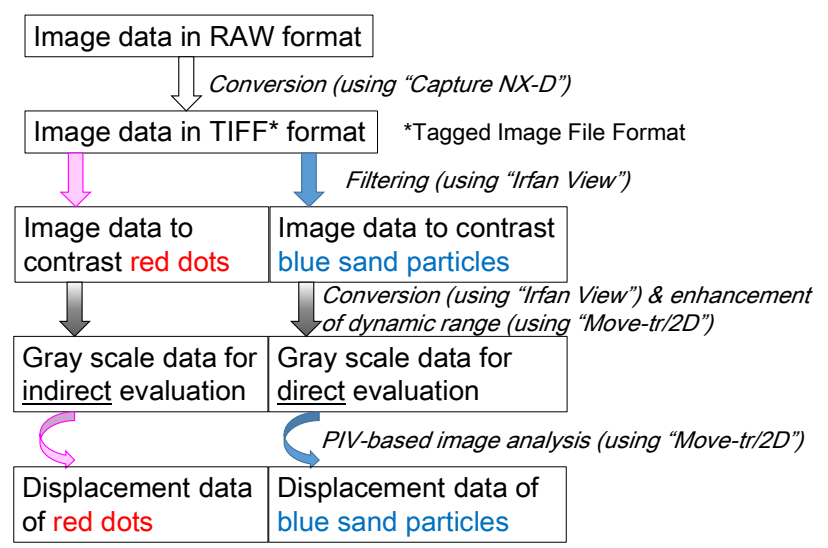

Fig. 2. Processing flow of image data.
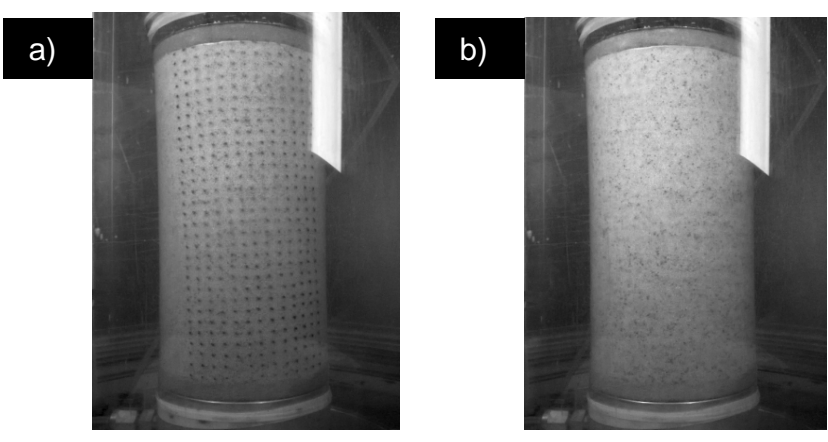

Fig. 3. Typical image after converting a) indirect and b) direct evaluation data into gray scale with dynamic range enhancement.
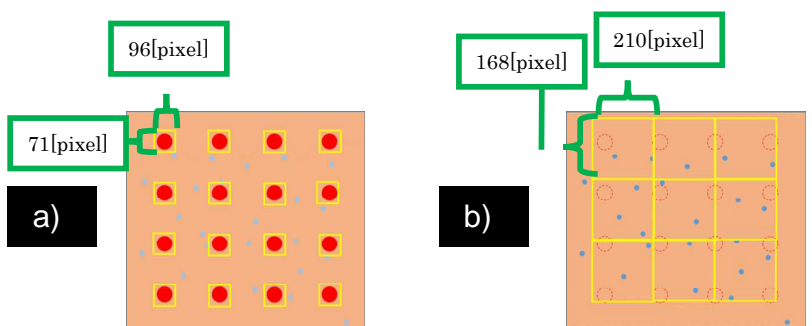

Fig. 4. Regions traced in PIV-based analyses; a) indirect evaluation and b) direct evaluation of full grid patterns.

\section{TEST RESULTS AND DISCUSSIONS}

\subsection{Undrained cyclic triaxial test results}

The behavior observed during the undrained cyclic loading is shown in Fig. 5 in terms of the effective stress path, stress-strain relationships and the time histories of excess pore water pressure ratio and the global axial strain computed based on the data measured with the external displacement transducer. 
The excess pore pressure ratio, defined as the ratio of excess pore water pressure to the initial effective stress $(=100 \mathrm{kPa})$, accumulated with the cyclic loading and reached unity, suggesting the occurrence of zero effective stress state or initial liquefaction. It was followed by gradual increase in the axial strain amplitude. After the double amplitude vertical strain exceeded $5 \%$ in the 11th cycle, the loading was terminated.
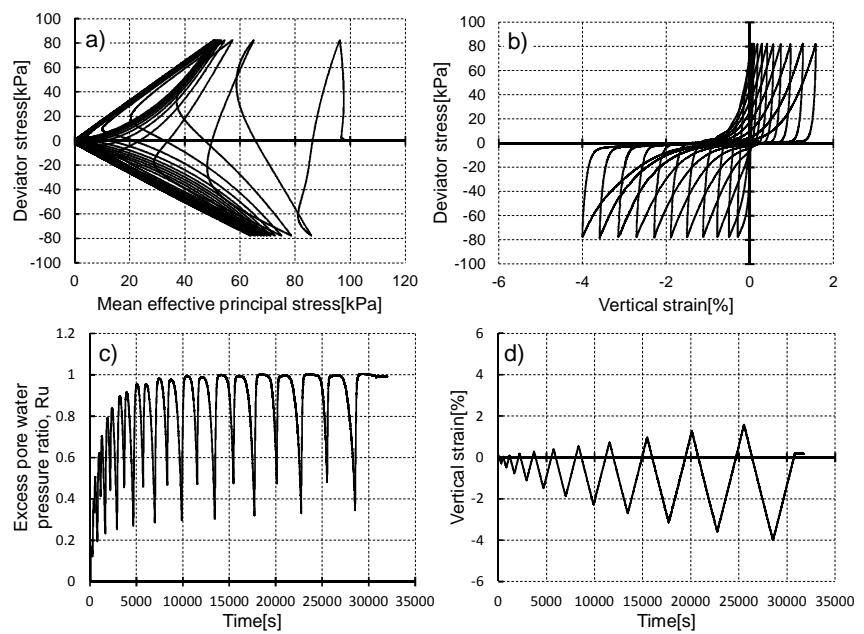

Fig. 5. Behavior observed during undrained cyclic loading; a) effective stress path, b) stress-strain relationships and time histories of c) excess pore water pressure ratio and d) global axial strain.

\subsection{Image analysis results}

The results from the image analyses to trace the red dots on the membrane (indirect evaluation) and the blue sand particles (direct evaluation of full grid patterns including the red dots) are compared in Fig. 6 in terms of the vertical displacements at nine regions that were arbitrary selected from the upper, middle, and lower rows and the left, central and right columns as shown in Fig. 7a. In general, good agreement could be obtained between the indirect and direct evaluation results.

Based on the distribution of vertical displacements obtained by the indirect evaluation and two kinds of direct evaluation, three sets of distribution of local vertical strains were computed. By selecting four characteristic states (i.e., the states when the vertical strain became the maximum and the minimum, and those when the excess pore pressure ratio exhibited the peak values, as defined in Fig. 7b), comparisons are made among the three sets of vertical strain distributions in Figs. 8 and 9 during 4th and 11th loading cycles where the DA value exceeded $1 \%$ and $5 \%$, respectively.

It can be seen from these figures that the distributions of the local vertical strain evaluated directly by the two methods were consistent with each other, suggesting that the existence of red dots on the membrane would not affect the direct observation results. In addition, results from indirect observation were also consistent with those from the above direct observations, except for the regions $\mathrm{A}$ through $\mathrm{C}$ as marked by using colored circles in Fig. 9. The results from the indirect observation by using red dots on the membrane in the regions near the top and bottom ends of the specimen (regions A and C in Fig. 9) may not be reliable, due to excessive deformation of the membrane near the bottom end that was filled by sand particles, and to possible formation of water film near the top end. The authors could not identify the reason for the exceptionally large local strain values observed in region $\mathrm{B}$ based on the direct observation excluding the red dots.

It should be noted that possible effect of the stratification that was created during the specimen preparation by compaction in ten layers using the wet-tamping method could be seen in Figs. 8 and 9, in which alternative formation of horizontal stripes appeared in the distributions of local vertical strain.
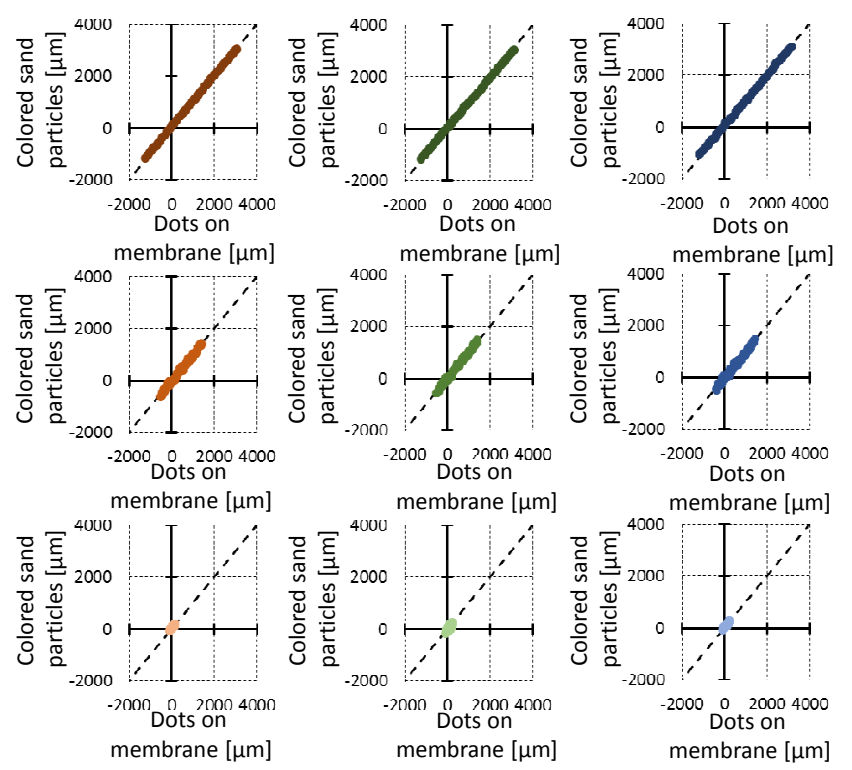

Fig. 6. Comparison of vertical displacements at selected nine points (see Fig. 7a for their location) obtained by tracing dots on membrane (indirect evaluation) and sand particles (direct evaluation of full grid patterns).

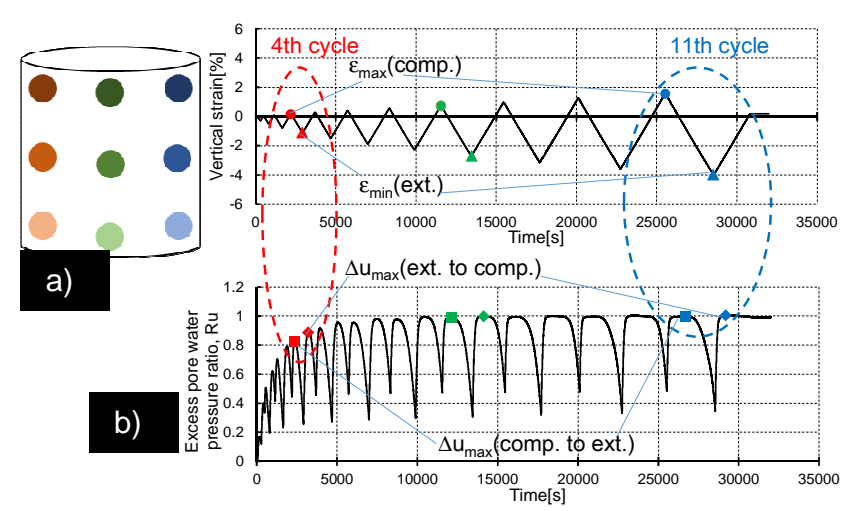

Fig. 7. a) Location of nine points analyzed in Fig. 6 and b) definition of characteristic states during 4th and 11th cycles for which distributions of local strain are compared in Figs. 8 and 9. 


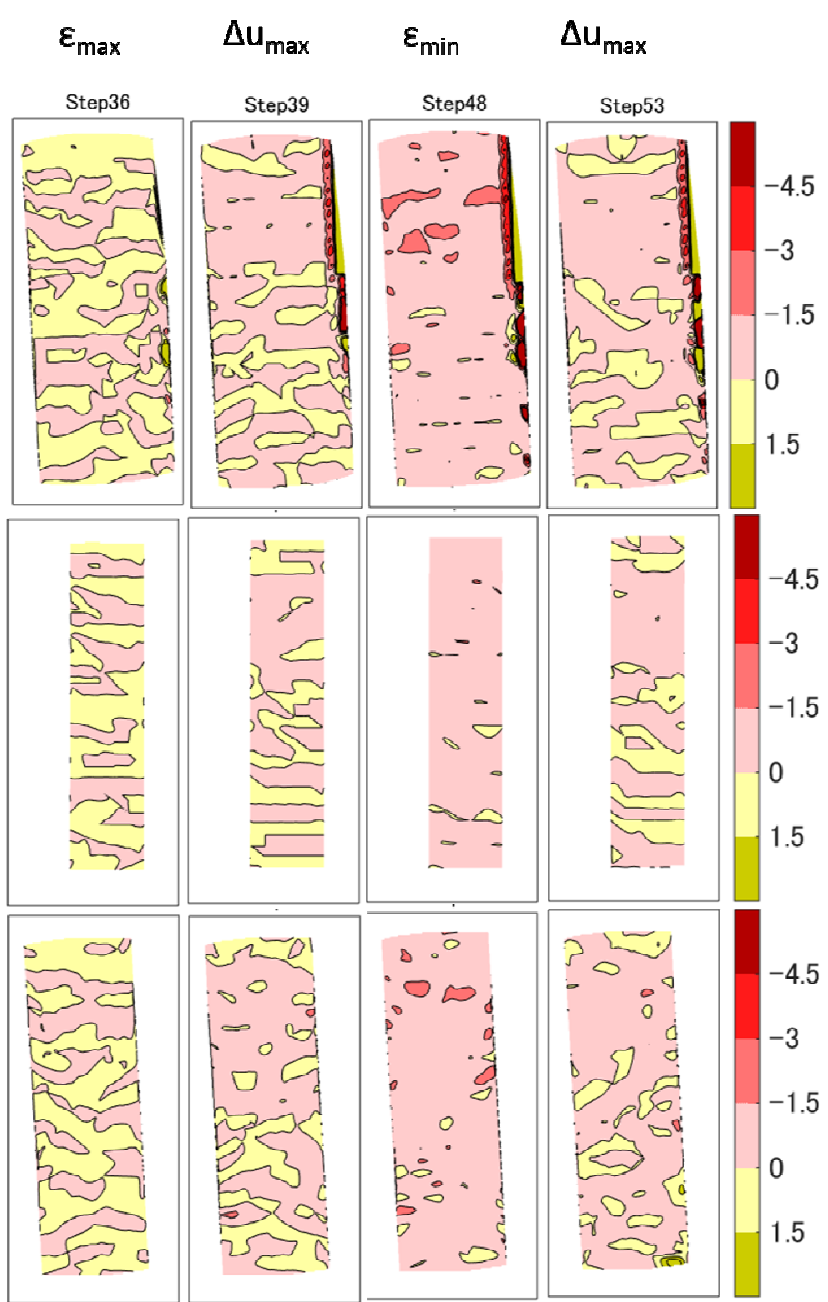

Fig. 8. Distributions of local vertical strain during 4th loading cycle; upper row by indirect evaluation, middle row by direct evaluation of full grid patterns and lower row by direct evaluation excluding dot regions.

\section{CONCLUSIONS}

A procedure to evaluate directly the local deformation properties of sand specimens was developed by using a transparent membrane and adding colored sand particles to the original ones. By combining it with indirect observation based on the image analysis of the membrane deformation, comparison of the two observations could be made on single specimen.

The local deformation properties of a dense Toyoura sand specimen evaluated by the indirect observation during the liquefaction process in undrained cyclic triaxial test up to a double amplitude axial strain of 5\% were consistent with those evaluated by the direct observation, except for the regions near the top and bottom ends of the specimen.

The results from the indirect observation of the regions near the top and bottom ends of the specimen may not be reliable, due possibly to formation of water film near the top end and excessive deformation of membrane near the bottom end

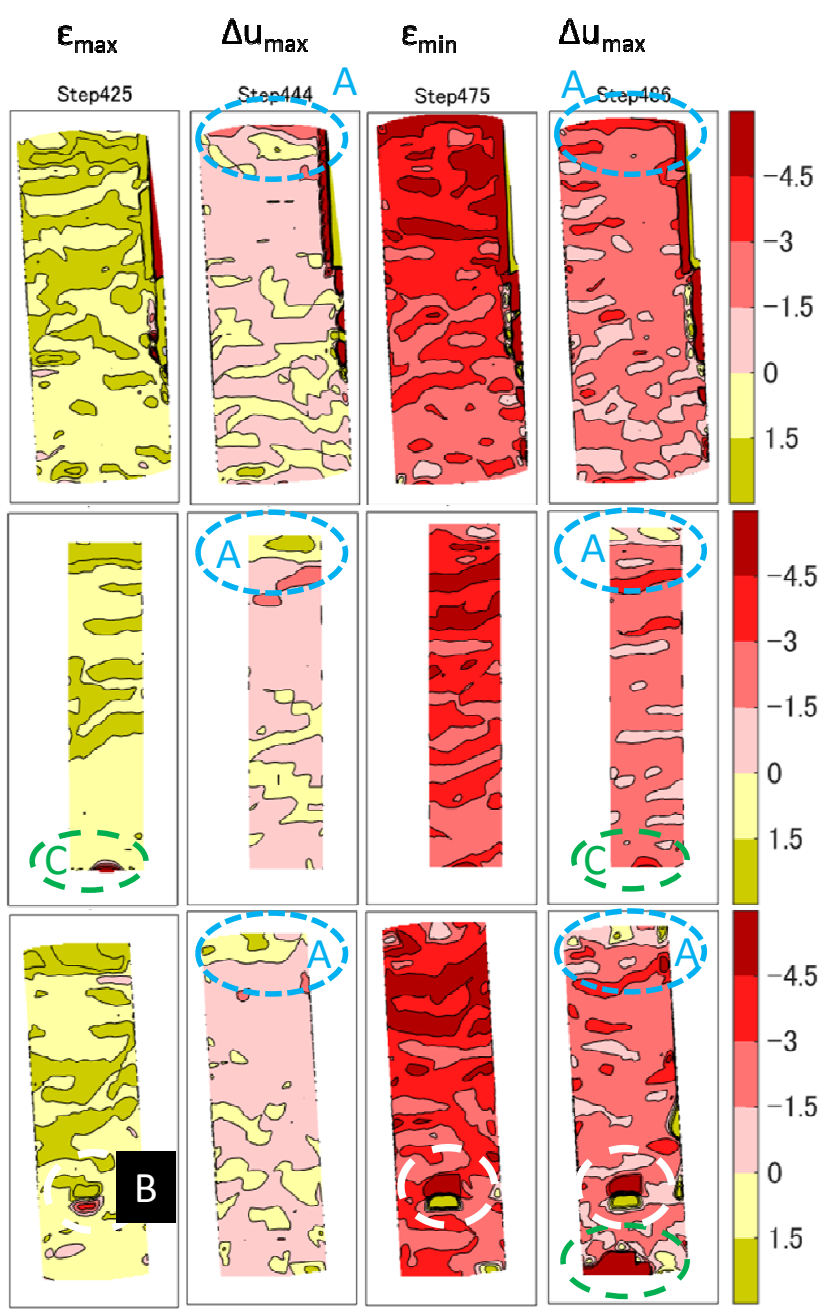

Fig. 9. Distributions of local vertical strain during 11th loading cycle; upper row by indirect evaluation, middle row by direct evaluation of full grid patterns and lower row by direct evaluation excluding dot regions.

\section{REFERENCES}

1) Ampadu, S.K. and Tatsuoka, F. (1993): "Effect of setting method on the behaviour of clays in triaxial compression from saturation to undrained shear", Soils and Foundations, 33(2), 14-34.

2) Fauzi, U.J., Wahyudi, S., Miyashita, Y. and Koseki, J. (2013): Image analysis technique for segregated specimen of Katori sand in hollow cylindrical torsional shear test, Proc. of 7 th International Joint Symposium on Problematic Soils and Geoenvironment in Asia, Okinawa, 163-166.

3) Ishimaru, M., Sawada, M., Koseki, J. and Miyashita, Y. (2011): "Failure behavior of artificial rock masses with discontinuities in plane strain compression tests", Journal of Japan Society of Civil Engineers, A2, 67(2), I_293-I_393 (in Japanese).

4) Rechenmacher, A. L. (2003): "Imaging-based experimental soil mechanics," Proceedings of the 1st Japan-US Workshop on Testing, Modelling and Simulation, ASCE, Geotechnical Special Publication, 143, 653-663.

5) Yoshida, T., Tatsuoka, F., Siddiquee, M. S. A., Kamegai, Y. and Park, C.S. (1994): "Shear banding in sands observed in plane strain compression", Localization and Bifurcation Theory for Soils and Rocks, Chambon, J Desrues, I. Vardoulakis (eds), Balkema, 165-179. 